\title{
Clinical Case of a 14-Year-Old Boy with Abdominal Stem-Shaped Twisted Necrotizing Vascular Malformation
}

\section{Yu Yu Sokolov' ${ }^{1}$, SV Stonogin ${ }^{2 *}$, EE Bibikova ${ }^{1}$, EA Tashirova ${ }^{2}$ and Zh R Omarova ${ }^{3}$}

${ }^{1}$ Russian Medical Academy of Continuing Postgraduate Education, Moscow, Russia ${ }^{2}$ Municipal Clinical Childrens Hospital Named After Z.A. Bashlyaeva, Moscow, Russia

${ }^{3}$ Children's State Hospital of St. Vladimir, Moscow, Russia

*Corresponding Author: SV Stonogin, Surgical Department, unicipal Clinical Childrens Hospital Named After Z.A. Bashlyaeva, Moscow, Russia.
Received: September 13, 2021

Published: September 23, 2021

(C) All rights are reserved by SV Stonogin., et al.

\begin{abstract}
Lymphatic malformations (LM) are benign neoplasms that form as a result of impaired formation of embryonic lymphatic sacs during embryogenesis.

A 14-year-old boy was admitted to the department of infectious diseases with complaints of fever, weakness, abdominal pain, sore throat and loose stools. Previously, the patient had complaints of abdominal pain for a year; ultrasound examination of the abdominal cavity was performed repeatedly, in which no pathological changes were detected. The patient underwent laparoscopic appendectomy; During the revision of the abdominal cavity, a space-occupying lesion emerging from the mesentery of the ascending colon and twisted around the vascular pedicle by 3 turns was revealed. Education removed. Histological and immunohistochemical examination confirmed the diagnosis of lymphatic-venous malformation.
\end{abstract}

Keywords: Lymphatic Malformation; Laparoscopy; Dysembryogenesis; Intra-Abdominal Malformation; Minimally Invasive Intervention; Immunohistochemical Study; Podoplanin; Abdominal Pain

\section{Introduction}

Lymphatic malformations (LM) are benign tumors originating from lymphatic vessels due to the dysembryogenesis starting from 6th week of perinatal period [17]. This condition is usually diagnosed at birth, but may be found at any age, including before birth via ultrasound investigation. Early diagnosis in older children is rather challenging. Prevalence of these conditions in neonates and infants is $1 / 60,000$ while in older children and adults it is lower
- 1/200,000 - 250,000; LM is more common in boys (3:1) [5]. Despite benign nature, LM have a trend toward infiltrating growth; they are characterized by frequent relapses and occasionally become infected. Clinical signs vary from asymptomatic course to the symptoms of "acute abdomen".

\section{Purpose of the Study}

We present very rare clinical case of abdominal stem-shaped twisted necrotizing vascular malformation in a 14-year-old boy. 


\section{Materials and Methods}

Fourteen-year-old boy was admitted to the department of infectious diseases with complaints on fever, weakness, abdominal pain, sore throat and liquid stool. According to the patient's words, he was followed-up at another healthcare institution with abdominal pain during one year. He underwent multiple abdominal ultrasound investigations, which did not show signs of tumor.

General condition of moderate severity. Body temperature: $37^{\circ} \mathrm{C}$. Poor appetite. Constitution is normal, with signs of overnutrition. Oropharyngeal hyperemia. Tonsils have signs of hypertrophy, edematous, with greyish cover in both lacunas. Abdomen has typical shape, not bloated. Soft. Tenderness in the right iliac area.

Blood test: high blood cell count: 10.6*109/l. Abdominal ultrasound showed significant accentuation of the vascular pattern of the liver. In the superior segment of the lateral channel, there is a retrocecal tubular aperistaltic structure with laminated walls, up to $20 \mathrm{~mm}$ in diameter, surrounded by echogenic tissues. Intestinal loops in the right part of the abdominal cavity are infiltrated, edematous, with thickened walls. Weal peristalsis in the affected segments. Free fluid: 86 × $39 \mathrm{~mm}$ above the bladder.

\section{Results}

This observation demonstrates a very rare pathological condition in children: intra-abdominal lymphatic malformation with torsion and necrosis. Laparoscopic surgery allowed to resect the lesion with minimum surgical injury.

\section{Conclusion: Echo-signs of acute appendicitis}

Urgent laparoscopy was performed. Upon revision it was noted that at the hepatic flexure greater omentum surrounds the round-shaped lesion (Figure 1). Greater omentum was separated using smooth tissue forceps. Upon revision it was noted that $5 \mathrm{x}$ $4 \times 3 \mathrm{~cm}$ dark-colored round-shaped tumor with cartilaginous density was twisted for 3 revolutions of $5 \mathrm{~cm}$ long thin pedicle ( $4 \mathrm{~mm}$ in diameter), which approaches greater omentum strand from above, and on the other side around thin $3 \mathrm{~cm}$ long pedicle ( $4 \mathrm{~mm}$ in diameter), which is directed to the mesenteric edge of the colon $25 \mathrm{~cm}$ from the ileocecal angle (Figure 2). Looped sutures (Polyglycolide 3.5 suture) was applied on the thin pedicle directed to the mesenteric edge of the colon. The pedicle was dissected with electrocautery. A segment of greater omentum was resected with electrocautery. Tumor was resected en block via 200 mm trocar.

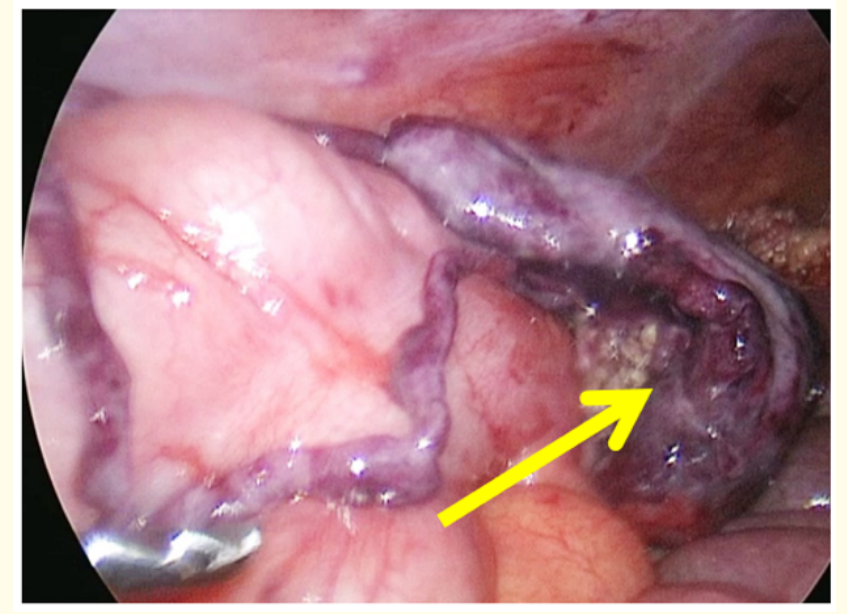

Figure 1: Intraoperative photograph - Dark stem-shaped tumor. Tumor pedicle originating from the greater omentum is visible, with torsion (Figure 3a, indicated by yellow arrow).

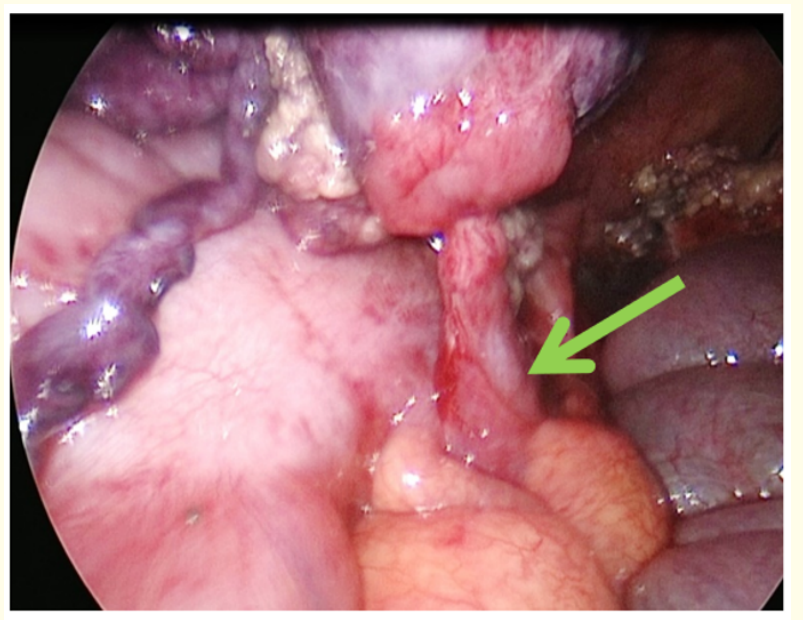

Figure 2: Intraoperative photograph - Short pedicle of the cystic lesion is visible at the ascending colon mesenteric edge (indicated by green arrow). 
On revision: Vermicular appendix is in small pelvis, extended up to $10 \mathrm{~mm}$, rigid. with signs of hyperemia. Length - $10 \mathrm{~cm}$. Small pelvis contains clear fluid in small pelvis. Lymph nodes in the mesenteric root are enlarged up to $15 \mathrm{~mm}$, with signs of hyperemia. Conclusion: acute appendicitis. Mesadennitis. Laparoscopic appendectomy was performed. Histopathological investigation showed signs of acute appendicitis.

In the postoperative period, the patient received antibacterial and antiviral therapy taking into account signs of infectious mononucleosis. Atypical mononuclear cells - 14\%; PCR of the saliva for herpesviruses of July 5 - Epstein-Barr virus was identified. Wounds were healed by primary intention. Sutures were removed on the 7 th day. The patient was discharged in satisfactory condition.

Tumor was cut open. Dimensions: $6.0 \times 5.0 \times 4.0 \mathrm{~cm}$, with 11.0 $\mathrm{cm}$ tubular structure. Internal and external surfaces have brownred color. Wall thickness $0.2-0.8 \mathrm{~cm} .3 .5 \times 3.0 \mathrm{~cm}$ omentum segment is yellow-brown, with congested vessels (Figure 3a, indicated by yellow arrow).

Taking into account partial tumor necrosis, major hemorrhages and lack of specific structures, unambiguous conclusion on the pathological process cannot be made, although the described histological pattern may occur in vascular malformation with secondary changes (Figure 3b). Immunohistochemical investigation showed that the lesion consists of multiple venous and lymphatic vessels of various size, with endothelial lining, without atypical features. There are areas of congested vessels, hemorrhages and necrosis with lysis and cystic cavities formation. Tumor wall is partially necrotized, with granular and fibrous tissue with multiple vessels of various diameter. They have thickened walls with dystrophic calcification, major hemorrhages. Infiltration with lymphocytes, histiocytes, eosinophils, neutrophils and plasmocytes was observed. External surface is partially covered with mesothelium, internal lining cannot be clearly identified. Intestinal wall elements were not found (Figure 3c). In the segment of omentum there are congested vessels, stasis, edema, hemorrhages, microfocal infiltration. Immunophenotyping: vascular endothelium is stained with CD34 и Vimentin, ki67 - low (<1\%) (Figure 3d).

\section{Discussion}

LM are attributed to the mature benign mesenchymal tumors. They comprise 9 - 10\% of all benign tumors in oncopediatrics [7].

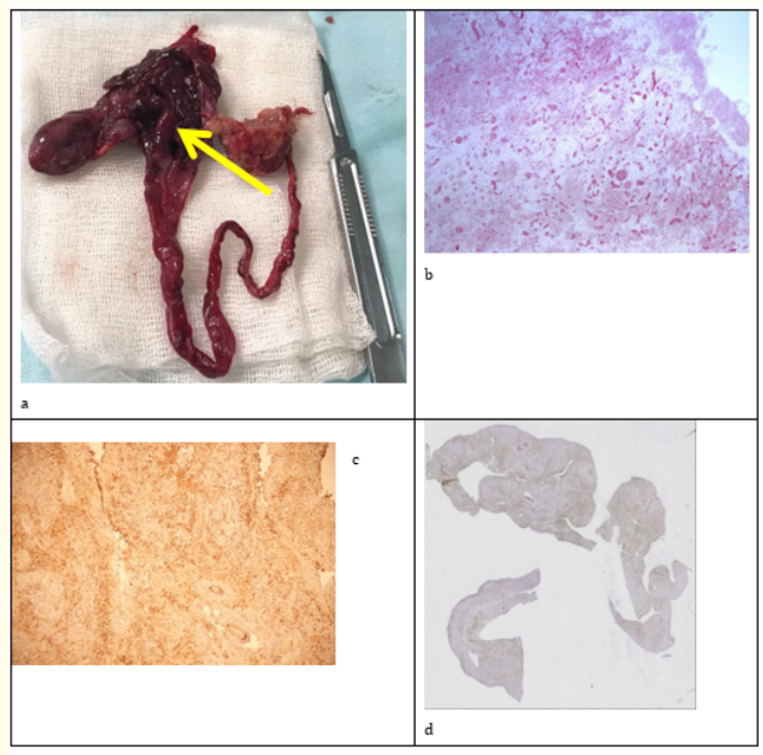

Figure 3: Figure 3a: Gross specimen of the resected tumor with a portion of the omentum. Figure $3 \mathrm{~b}$ : Histopathological investigation Microphotograph. Figure 3c: Immunophenotyping. Vascular endothelium is stained ith CD34 and Vimentin.

Morphological signs correspond to the lymphovenous malformation. Figure 3d: Immunophenotyping. Vascular endothelium is stained with ki67 - low $(<1 \%)$.

There are no published data on the signs of malignant transformation of these tumors. Although, despite benign nature, LM have a trend toward infiltrating growth; they are characterized by frequent relapses and occasionally become infected. Due to the genetic similarity of LM to the blood vessels, in particular, venous, curative surgical resection is challenging when the lesions are close to the major vessels, and residual lymphangiomatous tissue on the major vascular trunks may cause tumor relapses. This occurs in 6-7\% patients with LM [4].

A number of authors link LM with some syndromes, such as Klippel-Trenaunay syndrome, Nonne-Milroy disease etc [8].

According to the localization LM are categorized into superficial (LMs of the neck, limbs, trunk) and internal LMs. Internal localization of lymphangiomas and lymphohemangiomas (visceral 
forms) is uncommon, not more than $8-15 \%$ of all observed cases of lymphangiomas in children. Among visceral forms, pathological processes are more common in bowel and mesentery, spleen, omentum, liver, less common - in lymphatic vessels and mediastinal lymph nodes, as well as in the retroperitoneal fat. Most common localization is the mesentery of the small intestine (70\%). Among such forms, LM most often develop in the mesoileum 50 - 60\%) [7].

There is a documented case of lymphangioma involving inguinal canals and scrotum [1]. Other authors [6] described a clinical case of a child with LM of the bladder. There were cases of lymphatic malformation of the liver [21], which are characterized by pain syndrome, biliary obstruction or inferior vena cava syndrome.

According to the previous classification, there were 4 types of lymphangiomas: capillary (basic), cystic, cavernous and mixed (combination with hemangioma) $[3,18]$; currently, ISSVA classification is applied.

For diagnosis, combination of ultrasound and computed/ magnetic-resonance imaging (CT/MRI) is used. In complex cases, minimally invasive diagnostic surgeries are necessary. Immunohistochemical investigation is used for definitive diagnosis [5]. In such test endothelial cells show positive reaction with anti-podoplanin antibodies (Podoplanin; D2-40), and show variable expression with anti-FVIII-rAg, CD31 and CD34 antibodies [5].

Patients are categorized in three groups according to a number of specific clinical signs: First group - children with palpable tumor in the abdominal cavity. Second - patients admitted with signs of "acute abdomen" of bowel obstruction. Third group includes children with asymptomatic course $[4,14,15]$.

Lymphangiomas are characterized by a relatively favorable course, which, according to most authors, is due to their slow growth. Clinical signs in lymphangiomas of the mesentery of small and large intestine are variable and depend on the size, location, number of lymphatic cysts, relation to the intestine and adjacent organs, and complications [7].

Although, one should be aware of the complications which may be due to the tumor nature. Most common of them is inflammation. Infection usually occurs via lymphatic route [3]. Inflammation with suppuration of the lymphangioma contents is accompanied by a sharp deterioration of the child's general condition, fever and intoxication. Regional lymph nodes become enlarged and tender. In case of superficial localization of the lesion - signs of lymphangitis. Relapses are possible several months after the inflammation have resolved. There is a published description of a rare case of infection of the LM of the small intestinal mesentery root [3].

In another study, complications were documented in $33.3 \%$ patients of them infectious - in 6.3\%, and lymphorrhea in $27.1 \%$ [5]. Antibacterial therapy was applied for treatment of the infectious complications. $\mathrm{N}$ case of lymphorrhea, tactics of watchful waiting was applied up to 4 days. It has resolved without treatment during up to 5 days in $53.8 \%$ patients. For other children, it was decided to begin total parenteral nutrition. As a result, over the period from 4 to 9 days lymphorrhea have resolved almost in all cases [5].

There are two published cases of mesenteric lymphatic malformation in combination with malrotation [18]. In both presented cases malformations were adjacent to the duodenojejunal junction. Such location of malformation allowed authors to suppose that mesenteric lymphangioma in such cases might have developed as a rare acquired variant of the abnormality due to the chronic torsion [18]. Similar case of small intestine torsion with partial obstruction against the background of lymphatic malformation was described by Worapop Suthiwartnarueput., et al. Signs of infectious gastroenteritis in this patient might have possibly developed as a result of secondary inflammatory process due to the torsion, including hemorrhagic infarction [20].

Currently, there is no published consensus opinion on the benefits and indications for medical or surgical treatment.

Authors investigating the possibilities of therapeutic effect on lymphangioma tissues most commonly use such drug products as beta-blockers, glucocorticoids, monoclonal antibodies to vasculoendothelial growth factor (VEGF) - Bevacizumab, interferons, propranolol. New methods of treatment of lymphatic abnormalities are still emerging, taking into account high prevalence of such lesions and lack of therapeutic opportunities in complicated or relapsing lymphatic abnormalities. Recently, other novel products were introduced, of which most promising are Sildenafil, Propranolol and Sirolimus. Although there are no published data on their ability to significantly reduce tumor size.

If watchful waiting tactics or drug therapy are applied, it increases the risk of such complications as inflammation, suppura- 
tion, perforation, compression of the adjacent organs and tissues by the tumor, and lymphorrhea [3,5].

According to the published data, there is no generally accepted surgical treatment tactics. Various authors describe both minimally invasive organ sparing and curative methods. The use of combined minimally invasive surgical interventions (transcutaneous puncture, draining with selective embolization of vessel of the tumor wall, transcutaneous cyst puncture with deepithelization using 95\% ethanol, laparoscopic resection of the cyst roof with deepithelization using high-temperature plasma (PlasmaJet). If the cyst has challenging localization for laparoscopic manipulations, there is a description of one case of cyst puncture under ultrasound control $[2]$.

After percutaneous puncture with drainage of the lesion with deepithelization, in $4.1 \%$ cases authors noted infection of the residual cystic cavity with the need or repeated surgeries. On followup, relapses occurred in $6.2 \%$ children. In some children with partially resected lesions, relapsing lymphangiomas occurred due to the invasive growth through large neurovascular bundles. In such cases, gentle coagulation of the tumor surface was performed followed by surgical field draining [5]. In relation to one child, who underwent puncture of the cystic cavity with deepithelization, treatment had no effect [2].

According to a number of publications, sclerotherapy is effective for the treatment of gross cystic lymphatic abnormalities, and it is less effective in microcystic lymphatic abnormalities [8,19]. Usually, picibanil (OK-432), doxycycline, bleomycin, ethanol, sodium tetradecylsulfate, acetic acid and hypertonic sodium chloride solution are used as sclerosing agents. Chinese authors applied intrauterine sclerosing of the LM in fetuses with favorable results. Although, LMs were superficial [10].

Transcutaneous puncture with drainage and gradual sclerosis under laparoscopic control, can also be a safe method of treatment of splenic cysts. It can be applied repeatedly, but it does not exclude the risk of relapse [12].

Despite the cases of surgical treatment of visceral lymphangiomas are regularly discussed in literature, and various authors discuss the inappropriateness of aggressive surgical tactics, including wide resection of the adjacent tissues and organs [17], to date there is no clear trend towards transition to the application of minimally invasive videoendoscopic technologies for treatment of such patients. Partly, the reason is the rare nature of such observations.

When choosing surgical tactics, most authors look at the size and location of the lesion. According to Sharoev., et al. [7], it is reasonable or necessary to resect large spongious tumors via midline laparotomy, which enable sufficient revision of the abdominal cavity to assess intestinal patency as well as its blood supply. In infants, wide transverse laparotomy is possible. In cystic lesions and small tumors, laparoscopy can be employed [7].

Most authors indicate that decision making on the surgery tactics should be based on the multiple data analysis, including size and location of the cyst, and patient's age. Laparoscopic dissection of walls of the cystic cavity with to spare mesenteric vessels and adjacent intestinal segment [11]. Although, if it is necessary to resect a segment of the intestine in case of invasive tumor growth with anastomosis formation, classical laparoscopic approach should be avoided [9].

In the available literature, there are insufficient data on the results of treatment of the intraabdominal lymphatic malformations with the use of endovisual methods. The most significant challenges are due to anatomical features of the tumor, although magnification enabled by the modern endoscopic instruments and high-resolution optics allow to perform microsurgical tissue mobilization. Transabdominal approach enables the best room for surgical maneuver, freedom of movement and clear view on the anatomical landmarks. Main benefits versus open surgery are minimum injury to the anterior abdominal wall and short length of rehabilitation period [11]. According to Gunadi, total laparoscopic dissection of the LM is the best treatment method when the necessary equipment is available [13].

\section{Conclusion}

Currently, urgent laparoscopy is the most reliable method of diagnosis of the intra-abdominal pathological process in children. Laparoscopy allows to diagnose rare complicated developmental defects - torsion of the lymphatic malformation with necrosis.

\section{Bibliography}

1. VK Litovka., et al. "Large cystic lymphangioma of the retroperitoneal space and small pelvis with involvement of the inguinal canals and scrotum". Clinical Case 7.42 (2012): 127-129. 
2. Kh M Bataev., et al. "Organ sparing interventions in children with nonparasitic spleen cysts". Russian Bulletin of Pediatric Surgery, Anesthesiology and Resuscitation 7 (2017): 34-35.

3. II Babich and GL Shilov. "Complicated lymphangiomas of the mesentery root in a 12 year old child". Russian Bulletin of Pediatric Surgery, Anesthesiology and Resuscitation (2015): 75-76.

4. LV Angarkhaeva., et al. "First experience of endoscopic resection of the large retroperitoneal lymphangiomas in children". Siberian Journal of Medicine 4 (2015): 98-100.

5. IV Poddubny., et al. "Surgical treatment of lymphangiomas in children: clinical case series Oncopediarics 1 (2019): 53-64.

6. SM Bataev., et al. "Endosurgical treatment in children with lymphangiomas of rare localizations". Russian Bulletin of Pediatric Surgery, Anesthesiology and Resuscitation 7 (2015): 6869.

7. TA Sharoev., et al. "Lymphangiomas of the mesentery of the small intestine in children (literature review and own clinical observations)". Russian Bulletin of Pediatric Surgery, Anesthesiology and Resuscitation 2 (2012): 58-63.

8. BK Altyev., et al. "Lymphangioma of the small intestinal mesentery". Annals of the Emergency Medicine (2017): 69-73.

9. DA Morozov., et al. "Cystic lesions of spleen in children". Scientific Medical Journal of Saratov 7.3 (2011): 724-726.

10. MA Akselrov., et al. "Two cases of surgical treatment of the lymphangioma of the small intestine mesentery in newborns". Pediatric Surgery 21.5 (2017): 277-279.

11. YuYu Sokolov D.V., et al. "Surgical interventions in children with intraabdominal lymphangiomas". Russian Bulletin of Pediatric Surgery, Anesthesiology and Resuscitation 1 (2014): 2024.

12. Bagrodia N., et al. "Management of lymphatic malformations in children". Current Opinion in Pediatrics 27.3 (2015): 356-363.

13. Jiao-ling L., et al. "Fetal Lymphangioma: Prenatal Diagnosis on Ultrasound, Treatment, and Prognosis". European Journal of Obstetrics and Gynecology (2018): 1-18.
14. Gunadi Kashogi G., et al. "Pediatric patients with mesenteric cystic lymphangioma: A case series". International Journal of Surgery Case Reports 64 (2019): 89-93.

15. Soo-Hong K., et al. "Clinical features of mesenteric lymphatic malformation in children". Journal of Pediatric Surgery 51.4 (2016).

16. Otsubo Y., et al. "Small-Bowel Volvulus Caused By Mesenteric Lymphangioma". Clinical Gastroenterology and Hepatology (2019).

17. Chen J., et al. "Experience in the diagnosis and treatment of mesenteric lymphangioma in adults: A case report and review of literature". World Journal Gastrointestinal Oncology 10.12 (2018): 522-527.

18. Ousmane T., et al. "Cystic mesenteric lymphangioma: A case report". International Journal of Surgery Case Reports 61 (2019): 318-321.

19. Weeda VB., et al. "Mesenteric cystic lymphangioma: a congenital and an acquired anomaly? Two cases and a review of the literature". Journal of Pediatric Surgery 43 (2008): 1206-1208.

20. Capasso R., et al. "Lymphangioma of the small bowel mesentery in a 7-year-old girl: a report of a case investigated with ultrasonography, elastosonography and computed tomography". Journal Ultrasound 18 (2015): 297-300.

21. Suthiwartnarueput W., et al. "Lymphangioma of the small bowel mesentery: A case report and review of the literature". World Journal Gastroenterol 18.43 (2012): 6328-6332.

22. Budusan A., et al. "Isolated hepatic lymphangiomas in children: Two case reports". Journal of Pediatric Surgery Case Reports 56 (2020): 401-430.

\section{Volume 4 Issue 10 October 2021 (C) All rights are reserved by SV Stonogin., et al.}

